\title{
Novel Co-Channel Interference Signalling for User Scheduling in Cellular SDMA-TDD Networks
}

\author{
Rami Abu-alhiga ${ }^{1}$ and Harald Haas ${ }^{2}$ \\ The University of Edinburgh \\ United Kingdom
}

\section{Introduction}

The latest advancements of the $3^{\text {rd }}$ generation (3G) universal mobile telecommunications system (UMTS) have led to the long term evolution (LTE) standard release (referred to as 3.9G) within the $3^{\text {rd }}$ generation partnership project (3GPP). LTE does not meet the requirements for the fourth generation $(4 \mathrm{G})$ systems defined by the international telecommunication union (ITU). Therefore, work on LTE-Advanced within 3GPP has recently started. LTE-Advanced can be seen as the continuous evolution of wireless service provision beyond voice calls towards a true ubiquitous air-interface capable of supporting multimedia services (Sesia et al., 2009).

LTE-Advanced systems face a number of essential requirements and challenges which include coping with limited radio resources, increased user demand for higher data rates, asymmetric traffic, interference-limited transmission, while at the same time the the energy consumption of wireless systems should be reduced. Driven by the ever-growing demand for higher data rates to effectively use the mobile Internet, future applications are expected to generate a significant amount of both downlink (DL) and uplink (UL) traffic which requires continuous connectivity with quite diverse quality of service requirements. Given limited radio resources and various propagation environments, voice over IP applications, such as Skype, and self-generated multimedia content platforms, such as YouTube, and Facebook, are popular examples that impose a major challenge on the design of LTE-Advanced wireless systems. One of the latest studies from ABI Research, a market intelligence company specializing in global connectivity and emerging technology, shows that in 2008 the mobile data traffic around the world reached 1.3 Exabytes $\left(10^{18}\right)$. By 2014, the study expected the amount to reach 19.2 Exabytes. Furthermore, it has been shown that video streaming is one of the dominating application areas which will grow significantly (Gallen, 2009).

In order to meet such diverse requirements, especially, the ever-growing demand for mobile data, a number of different technologies have been adopted within the LTE-Advanced framework. These include smart antenna (SA)-based (also known as directional antennas or antenna arrays) multiple-input multiple-output (MIMO) systems (Bauch \& Dietl, 2008a;b; Foschini \& Gans, 1998; Kusume et al., 2007) and efficient multiuser transmission techniques such as multiuser MIMO using precoding to achieve, for example, space division multiple access (SDMA) (Fuchs, et al., 2007), and networked MIMO, i.e. coordinated multipoint (CoMP) systems. Therefore, there is a broad agreement recently among LTE standardization groups 
that MIMO will be the key to achieve the promised data rates of 1 Gbps and more (Seidel, 2008).

It is well known that co-channel interference (CCI), caused by frequency reuse, is considered as one of the major impairments that limits the performance of current and $4 \mathrm{G}$ wireless systems (Haas \& McLaughlin, 2008). To outmaneuver such obstacle, various techniques such as joint detection, interference cancelation, and interference management have been proposed. One of the most promising technology is to utilize the adaptability of SAs. Spatial signal pre-processing along with SAs can provide much more efficient reuse of the available spectrum and, hence, an improvement in the overall system capacity. This gain is achievable by adaptively utilizing directional transmission and reception at the base station (BS) in order to enhance coverage and mitigate CCI. One of the key challenges to overcome, however, is the signalling overhead which increases drastically in MIMO systems.

Unlike the traditional resource allocation in single-input single-output (SISO) fading channels, which is performed in time and frequency domains, the resources in MIMO systems are usually allocated among the antennas (the spatial domain). From closed-loop MIMO point of view, channel aware adaptive resource allocation has been shown to maintain higher system capacity compared to fixed resource allocation (Ali et al., 2007; Gesbert et al., 2007; Koutsimanis \& Fodor, 2008). In particular, adaptive resource allocation is becoming more critical with scarce resources and ever-increased demand for high data rates.

It is shown that for closed-loop MIMO the optimal power allocation among multiple transmit antennas is achieved through the water-filling algorithm (Telatar, 1999). However, to enable optimal power allocation, perfect channel state information (CSI) at the transmitter is required. Some other work focused on transmit beamforming and precoding with limited feedback (Love, et al., 2005; 2003; Mukkavilli et al., 2002; 2003; Zhou et al., 2005), where the transmitter uses a quantized CSI feedback to adjust the power and phases of the transmitted signals. To further reduce the amount of feedback and complexity, different strategies such as per-antenna rate (an adaptive modulation and coding approach that controls each antenna separately) and power control algorithms have been proposed (Catreux et al., 2002; Chung et al., 2001a;b; Zhou \& Vucetic, 2004; Zhuang et al., 2003). By adapting the rate and power for each antenna separately, the performance (error probability (Gorokhov et al., 2003) or throughput (Gore et al., 2002; Gore \& Paulraj, 2002; Molisch et al., 2001; Zhou et al., 2004)) can be improved greatly at the cost of slightly increased complexity. Additionally, antenna selection is proposed to reduce the number of the spatial streams and the receiver complexity as well. Various criteria for receive antenna selection or transmit antenna selection are presented, aiming at minimizing the error probability (Bahceci et al., 2003; Ghrayeb \& Duman, 2002; Gore et al., 2002; Gore \& Paulraj, 2002; Heath \& Paulraj, 2001; Molisch et al., 2003) or maximizing the capacity bounds (Molisch et al., 2003; Zhou \& Vucetic, 2004). It is shown that only a small performance loss is experienced when the transmitter/receiver selects a good subset of the available antennas based on the instantaneous CSI (Zhou et al., 2004). However, it is found that in spatially correlated scenarios, proper transmit antenna selection cannot just be used to decrease the number of spatial streams, but can also be used as an effective means to achieve multiple antenna diversity (Heath \& Paulraj, 2001). When the channel links exhibit spatial correlation (due to the lack of spacing between antennas or the existence of small angular spread), the degrees of freedom (DoF) of the channel are usually less than the number of transmit antennas. Therefore, using transmit antenna selection, the resources are allocated only to the uncorrelated spatial streams so that an enhanced capacity gain can be achieved. 
Most of the above work focused on the point-to-point (P2P) link in single user scenario. In a multiuser MIMO (MU-MIMO) context, MIMO communication can offer significant capacity growth by exploiting spatial multiplexing and multiuser scheduling. Therefore, opportunistic approaches have recently attracted considerable attention (Choi et al., 2006; Viswanath et al., 2002). So far, opportunistic resource allocation in a MU-MIMO scenario is still an open issue. Wong et al. and Dai et al. (Dai et al., 2004; Wong et al., 2003) consider a multiuser MIMO system and focused on multiuser precoding and turbo space-time multiuser detection, respectively. More recent work has addressed the issue of cross-layer resource allocation in DL MU-MIMO systems (Wang \& Murch, 2005). In broadcast MU-MIMO channels, dirty-paper coding (DPC) (Costa, 1983) can achieve the maximum throughput (Goldsmith et al., 2003; Vishwanath et al., 2003; Weingarten et al., 2004). In particular, DPC can accomplish this by using successive interference precancelation through employing complex encoding and decoding. Unfortunately, DPC is classified as a nonlinear technique that has very high complexity and is impractical. Due to the fact that DPC is computationally expensive for practical implementations, its contribution is primarily to determine the achievable capacity region of MU-MIMO channel under a per-cell equal power constraint. Therefore, many alternative practical precoding approaches are proposed to offer a trade-off complexity for performance (Airy et al., 2006; Chae et al., 2006; Hochwald et al., 2005; Pan et al., 2004; Shen et al., 2005; Windpassinger et al., 2004). These alternatives considered different criteria and methods such as minimum mean squared error (MMSE) (Schubert \& Boche, 2004; Shi et al., 2008), channel decomposition, and zero forcing (ZF) (Chen et al., 2007; Choi \& Murch, 2004; Spencer et al., 2004; Wong et al., 2003).

One of the most attractive approaches is the block diagonalization (BD) algorithm which supports orthogonal multiple spatial stream transmission. In BD algorithm, the precoding matrix of each user is designed to lie in the null space of all remaining channels of other in-cell users, and hence the intracell multiuser CCI is pre-eliminated (Chen et al., 2007; Shen et al., 2005; Spencer et al., 2004). In particular, SA-based SDMA, implementing BD algorithm, can multiplex users in the same radio frequency spectrum (i.e. same time-frequency resource) within a cell by allocating the channel to spatially separable users. This can be done while maintaining tolerable, almost negligible, intracell CCI enabled by BD signal pre-processing capabilities. Moreover, channel aware adaptive SDMA scheme can be achieved through joint exploitation of the spatial DoF represented by the excess number of SAs at the BS along with multiuser diversity. Generally, the radio channel encountered by an array of antenna elements is referred to as beam. In other words, SA technology along with BD algorithm can enable the BS to adaptively steer multiple orthogonal beams to a group of spatially dispersed mobile stations (MSs) (Choi et al., 2006), as depicted in Fig. 1.

The joint beam selection and user scheduling for orthogonal SDMA-TDD (time division duplex) system is a key problem addressed in this chapter. From precoding point of view, the availability of CSI of all in-cell users at the BS is crucial in multiuser (MU)-MIMO communication scenario to optimally incorporate different precoding techniques such as BD, adaptive beamforming, or antenna selection, in order to increase the overall system spectral efficiency. Basically, there are two methods for providing a BS with CSI of all associated MSs, namely limited (quantized) feedback and analog feedback. Limited feedback (also know as direct feedback) involves the MS to measure the DL channel and to transmit a feedback messages of quantized CSI reports to the BS during the UL transmission. Alternatively, the second method, referred to as UL channel sounding according to LTE terminology, involves the BS to estimate the DL channel based on channel response estimates obtained 


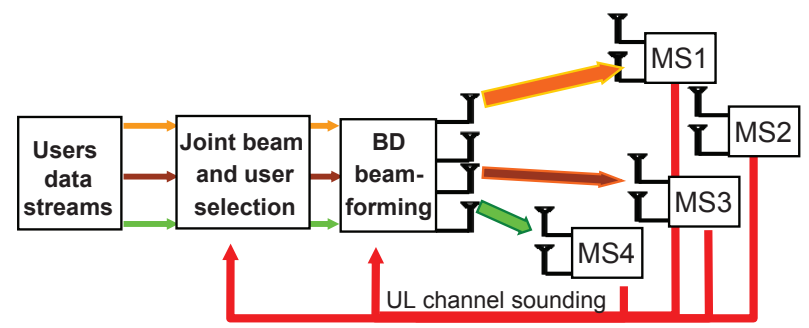

Fig. 1. A block diagram of SA-based MU-MIMO transmission implementing BD beamforming

from reference signals (pilots) received from the MS during UL transmission. Channel sounding offers advantages in terms of overhead, complexity, estimation reliability, and delay. Closed-loop SDMA-TDD networks can benefit from these advantages to avoid outdated feedback scenarios, enhance the network throughput, and reduce the computational cost at the user side. Clearly, TDD systems offer a straightforward way for the BS to acquire the CSI enabled through channel reciprocity (Love, et al., 2004). The advantages of UL channel sounding are discussed later in this chapter and a more detailed treatment can be found, for instance, in the technical documents of the evolved universal terrestrial radio access (EUTRA) study item launched in the LTE concept (Sesia et al., 2009).

In summary, UL channel sounding method is considered as one of the most promising feedback methods for SA-based SDMA-TDD systems due to its bandwidth and delay efficiency. In particular, UL channel sounding avoids the usage of dedicated feedback physical channels which results in utilizing the available bandwidth for data transmission much more effectively. In addition, UL channel sounding requires a shorter duration of time to convey the feedback information to the BS compared to the direct feedback method. This feature reduces the probability of having outdated feedback especially in fast varying channel conditions.

In interference-limited scenarios and according to Shannon capacity formula, the system performance is limited by the CCI from adjacent cells. Meanwhile, conventional channel sounding (CCS) only conveys the channel state information (CSI) of each active user to the BS. Therefore, CSI is only a suboptimal metric for multiuser spatial multiplexing optimization in interference-limited scenarios.

In light of the above, the benchmark system considered in this chapter for the system level analysis of the feedback methodology is a closed-loop SDMA TDD system. In the benchmark system, a BD technique is utilized to optimize the MU-MIMO spatial resources allocation problem based on perfect instantaneous CSI of each in-cell active user obtained from UL channel sounding pilots. The main goal of the benchmark system is to adaptively communicate with a group of users over disjoint spatial streams while optimizing the gains of the MU-MIMO channels. The optimization aims at enhancing the overall system capacity using fixed and uniformly distributed transmit power.

Most of the ZF-based precoding algorithms (e.g. BD) have been designed to only mitigate intracell CCI from different users in the same cell without considering CCI coming from transmitters in neighbouring cells. In a cellular environment, especially when full frequency reuse is considered, intercell (also known as other-cell) CCI becomes a key challenge which cannot be eliminated by BD-like algorithms. Moreover, it is shown that intercell CCI can significantly degrade the performance of SDMA systems (Blum, 2003). More specifically, if the 
BS schedules a group of users only based on the available CSI, the scheduling decision may be optimum for a noise limited system, but high intercell CCI at the respective MSs might render the scheduling decision greatly suboptimum. Therefore, the signal-to-interference-plus-noise ratio (SINR) would be a more appropriate metric in multicell interference limited scenarios, but this metric cannot directly be obtained from CCS. Thus, the key challenge here is to provide knowledge of intercell CCI observed by each user to the BS in addition to CSI. If, furthermore, intercell CCI observed by each SA at the BS itself is taken into account, the beam selection and user scheduling process can be jointly improved for both DL and UL (Abualhiga \& Haas, 2008).

\section{Contributions and assumptions}

The contribution associated with the feedback-based interference management for SDMA presented in this chapter can be split into three main parts:

- A novel interference feedback mechanism is developed. Specifically, it is proposed to weight the UL channel sounding pilots by the level of the received intercell CCI at each MS. The weighted uplink channel sounding pilots act as a bandwidth-efficient and delay-efficient means for providing the BS with both CSI and intercell CCI experienced at each active user. Such modification will compensate for the missing interference knowledge at the BS when traditional UL channel sounding is used. In addition, through exploitation of channel reciprocity the technique will act as implicit inter-cell interference coordination (ICIC) avoiding any additional signalling between cells.

- A novel procedure is developed to make the interference-weighted channel sounding (IWCS) pilots usable for the scheduler to optimize the spatial resource allocation during the UL slot. It is proposed to divide the metric obtained from the IWCS pilots by the intercell CCI experienced at the BS. The resulting new metric, which is implicitly dependent on DL and UL intercell CCI, provides link-protection awareness and it is used to jointly improve spectral efficiency in UL as well in DL.

- Finally, in order to facilitate a practical implementation, a heuristic algorithm (HA) is proposed to reduce the computational complexity to solve user scheduling problem.

The key assumptions for the system level analysis of the IWCS pilots performance can be summarized as follows:

- The considered closed-loop SDMA system enjoys perfect knowledge of the MIMO channel coefficients of each active user. Hence, this channel knowledge at both BS and MS is exploited to decompose the channel matrix into a collection of uncoupled parallel SISO channels.

- The considered problem of jointly adapting the MU-MIMO link parameters for a set of flat fading co-channel interfering MIMO links exploits two DoF: transmit antenna selection, and user selection. Since these two DoF are associated with two different layers (the physical (PHY) layer and medium access control (MAC) layer) the problem is considered to be optimized in a cross-layer fashion.

- The time and frequency DoF (e.g. frequency channel dependent scheduling and dynamic frequency resource allocation) are not considered in this study.

- This chapter assumes that appropriate methods are in place that completely eliminate or avoid intracell CCI. Therefore, the system is only limited by intercell CCI. However, 
the level of intercell CCI usually outweighs thermal noise and the system is, therefore, interference limited.

- According to the definition of the UL channel sounding mechanism adopted by LTE, channel sounding pilots are different from the demodulation pilots dedicated to the process of coherent data detection. This implies that modifying the UL channel sounding pilots does not hamper the channel estimation processes required for coherent data detection. In particular, the only purpose of the proposed modification on the UL channel sounding pilots is to add interference awareness to the channel sounding technique. In addition, according to the LTE technical documents related to the UL channel sounding pilots, the predetermined sounding waveforms are transmitted using orthogonal signals among all active users in all cells using the same frequency band. The sounding pilot sequences are chosen to be orthogonal in frequency domain among all of the users' antennas (Sesia et al., 2009). In summary, the above properties enable the BS to estimate the UL wideband channel for each antenna of each active user without any intracell or intercell CCI between the channel sounding pilots. Moreover, errors in the channel estimation due to the presence of noise is beyond the scope of this chapter. As a consequence, perfect channel estimation is considered as outlined in the first assumption.

\section{Overview of feedback methods}

Basically, there are two methods for providing a BS with the CSI of all MSs, namely direct feedback and UL channel sounding.

1. Direct feedback: According to LTE terminology this feedback method is termed codebook-based feedback (Abe \& Bauch, 2007). The MS determines the best entry in a predefined codebook of precoding (beamforming) vectors/matrices and transmits a feedback indication to the BS conveying the index value. In codebook feedback, the MS uses downlink channel estimates to determine the best codebook weight or weights for the BS to use as a precoding vector/matrix. The MS creates a feedback indication that includes the codebook index and then sends the feedback indication to BS. This method can be considered as a candidate option for frequency division duplex (FDD) systems which require an explicit transfer of the DL CSI during the UL transmission due to the absence of channel reciprocity.

It is worth mentioning that the physical feedback channel needs to have some reference signals to facilitate the coherent detection of the feedback information at the BS.

2. UL channel sounding: The MS transmits a sounding waveform on the UL and the BS estimates the UL channel of the MS from the received sounding waveform. The sounding pilot sequences are chosen to be orthogonal between all of the users' antennas and also are designed to have a low peak to average power ratio (PAPR) in the time domain, (Fragouli, et al., 2003; Popovic, 1992). The details of UL channel sounding are given in 3GPP technical documents (Sesia et al., 2009). However, a brief treatment of the uplink channel sounding signal model is given below.

According to LTE technical documents related to uplink channel sounding, the BS instructs the MS where and how to sound (i.e., send a known pilot sequence) on the uplink. The information obtained from uplink channel sounding at the BS is used to determine DL beamforming weights for MIMO channel dependent scheduling on the uplink, as well as for MIMO channel dependent scheduling on the DL. According to the structure discussed 
in 3GPP technical documents, channel sounding pilots enable the BS to estimate the UL wideband channel for each antenna of each active user without any intracell or intercell CCI between the channel sounding pilots.

Any codebook-based feedback scheme must account for the number of SAs at the BS. In a codebook-based feedback scheme, the MS must be able to estimate the DL channel no matter how many SAs are available at the BS. Thus, the computational complexity at the MS, and the information that is required to be fed back increase with the number of antennas at BS. In contrast, channel sounding schemes are independent of the number of BS antennas. In other words, the problem of channel estimation is much more difficult in a codebook-based feedback scheme than in a channel sounding scheme. More specifically, in codebook-based feedback, the air-interface must enable the MS to estimate the channel between its antennas and a relatively larger number of SAs at the BS. Such estimation imposes a heavy processing load on a MS in a direct feedback scheme, while in a channel sounding scheme the estimation process takes place at the BS side (Hassibi \& Hochwald, 2003).

For instant, consider the case where the BS has eight transmit antennas and the MS has a two receive antennas. In a channel sounding scheme, the BS must estimate the channel between its eight antennas and the two transmit antennas. In contrast, in a codebook-based feedback scheme, the air interface must enable the MS to estimate the channel between its two antennas and the eight transmit antennas (an eight-source channel estimation problem, which is much more difficult).

In TDD systems codebook-based feedback schemes tend to have much higher latency between the time of the channel estimation and the time of the subsequent DL transmission. The resulting outdated CSI can have detrimental effects on the performance of closed-loop transmission schemes, especially in fast fading channels. In contrast, channel sounding reference signals can be transmitted at the end of the UL slot. They can directly be exploited for the subsequent DL transmission. For these reasons, in a TDD system, UL channel sounding is preferred over codebook-based feedback.

\section{SDMA with block diagonalization adaptive beamforming}

\subsection{Overview of SA technology}

Originally, SA pre-processing techniques were proposed for military communications. Due to the significant technological advancements over the past two decades, SA-based technologies have become a cost-efficient solution for commercial communication systems to overcome some of the major challenges such as multipath fading, CCI, and capacity limitations especially for the cell-edge users. By exploiting the spatial diversity and the spatial processing capabilities of SA, an efficient utilization of available bandwidth and, hence, an increased system spectral efficiency is facilitated.

This section highlights the major features of SA-based SDMA systems relevant for the main contributions in this chapter. More specifically, the review is aimed at the benchmark SDMA system considered in this chapter. Also, this section briefly describes the generalized BD beamforming method for multiuser SDMA system (Pan et al., 2004), where the BS transmits multiple spatially multiplexed independent data streams to a group of users selected according to a scheduling criterion. Due to physical size constraints at the user side, the MSs are assumed to be equipped with limited number of multiple omnidirectional antennas (OAs) (two throughout this chapter). This assumption is also convenient in order to maintain affordable cost and reduced complexity at the mobile. As depicted in Fig. 2, each SA 


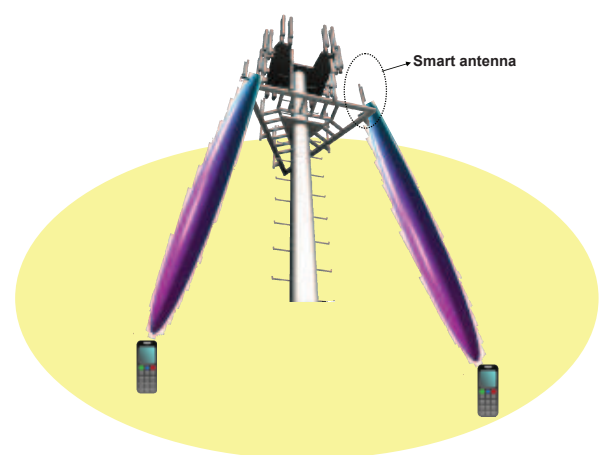

Fig. 2. A schematic illustration of a SA-based beamforming SDMA system

consists of an array of antenna elements and is dedicated to directionally transmit/recieve a single independent spatial stream. Such spatial stream is referred to as an effective antenna according to LTE terminology.

\subsection{Multiuser MIMO BD system model}

Consider a single-cell downlink MU-MIMO system where the BS is equipped with $N_{T}$ transmit SAs, and communicates over multiple MIMO channels with $K$ users. It is assumed that all users are equipped with the same number of receiving OAs denoted as $N_{R}$. To simplify the following analysis, it is assumed that $N_{R}<N_{T}$ and $N_{T}$ is an integer multiple of $N_{R}$ in order to serve all users. Fig. 3 illustrates an example of the considered SDMA system where $N_{R}=2$.

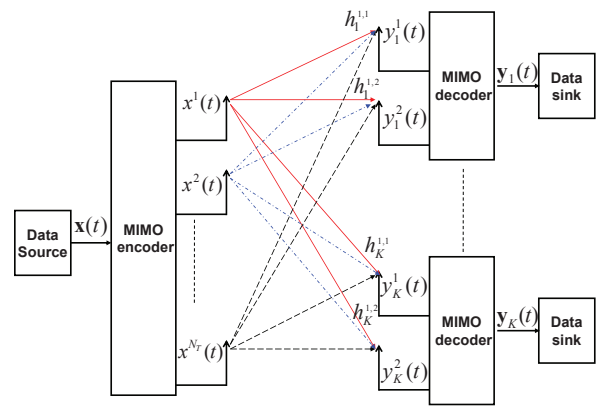

Fig. 3. SDMA system model with multiple MIMO users each equipped with 2 OAs

The flat fading MIMO channel matrix for user $k$ is denoted as $\mathbf{H}_{k}$ where $h_{k}^{(j, i)}$ is the fading coefficient between the $j^{\text {th }}$ transmit antenna and the $i^{\text {th }}$ receive antenna of user $k$. For analytical simplicity, the rank $r_{k}$ of $\mathbf{H}_{k}$ is assumed to be equal to $\min \left(N_{R}, N_{T}\right)$ for all users. Again, channel estimation errors caused by various reasons such as feedback delay, and feedback quantization error, etc. are beyond the scope of this chapter. Hence, it is assumed that the BS has perfect CSI for all users. By assuming that the number of data streams $s_{k}$ to user $k$ is equal to $N_{R}$, the transmitted data streams to user $k$ can be denoted as a $N_{R}$-dimensional vector $\mathbf{x}_{k}$ where $\sum_{k=1}^{K} s_{k} \leq N_{T}$. Since CSI is available at both sides of the MIMO link, it is assumed that the MIMO transmission includes linear pre-processing and post-processing performed at 
both BS and MS, respectively. Prior to transmission, the data vector of user $k, \mathbf{x}_{k}$, is multiplied by a $N_{T} \times N_{R}$ precoding matrix $\mathbf{T}_{k}$. In this chapter, it is assumed that $\mathbf{T}_{k}$ is generated using the $\mathrm{BD}$ beamforming algorithm which is a member of the zero-forcing (ZF) type multiuser precoding algorithms (Spencer et al., 2004). At the BS, the data vectors of the $K$ users are linearly superimposed and propagated over the channel from all $N_{T}$ antennas simultaneously. It is also assumed that the elements of $\mathbf{x}_{k}$ are independent and identically distributed (i.i.d.) with zero mean and unit variance. The signal vector received at user $k$ is

$$
\mathbf{y}_{k}=\mathbf{H}_{k} \sum_{l=1}^{K} \mathbf{T}_{l} \mathbf{x}_{\iota}+\mathbf{n}_{k}
$$

Equation (1) can be rewritten in the form of summation of the desired signal, the interference signal, and the noise signal as follows

$$
\mathbf{y}_{k}=\mathbf{H}_{k} \mathbf{T}_{k} \mathbf{x}_{k}+\mathbf{H}_{k} \sum_{\iota \neq k}^{K} \mathbf{T}_{\iota} \mathbf{x}_{\iota}+\mathbf{n}_{k}
$$

where the first term on the right-hand-side of (2) is the desired signal, the second term denotes the intracell CCI experienced by user $k$, and the last term $\mathbf{n}_{k}$ is the additive white Gaussian noise vector received by user $k$. According to key principles of BD algorithms, $\mathbf{T}_{\iota}$ is designed such that $\mathbf{H}_{k} \mathbf{T}_{\iota}=0$ for $\forall \iota \neq k$ and , hence, the intracell CCI is completely eliminated (nulled). The block matrices $\mathbf{H}_{\mathrm{S}}$ and $\mathbf{T}_{\mathrm{S}}$ can be defined as the system channel matrix and the system precoding matrix, respectively, as follows:

$$
\begin{gathered}
\mathbf{H}_{\mathrm{S}}=\left[\mathbf{H}_{1}^{H} \mathbf{H}_{2}^{H} \ldots \mathbf{H}_{K}^{H}\right]^{H} \\
\mathbf{T}_{\mathbf{S}}=\left[\mathbf{T}_{1} \mathbf{T}_{2} \ldots \mathbf{T}_{K}\right]
\end{gathered}
$$

Under the constraint of zero intracell CCI among users within the same cell, the optimal solution can be obtained by diagonalizing the product of (3) and (4) $\mathbf{H}_{\mathrm{S}} \mathbf{T}_{\mathrm{S}}$. Now, $\tilde{\mathbf{H}}_{S}$ can be defined as the block matrix of the MIMO links interfering with user $k$ as follows

$$
\tilde{\mathbf{H}}_{S}=\left[\tilde{\mathbf{H}}_{1}^{H} \ldots \tilde{\mathbf{H}}_{k-1}^{H} \tilde{\mathbf{H}}_{k+1}^{H} \ldots \tilde{\mathbf{H}}_{K}^{H}\right]^{H}
$$

In light of the above, the intracell CCI free constraint requires that $\mathbf{T}_{k}$ is selected to lie in the null space of $\tilde{\mathbf{H}}_{S}$. The details of designing the BD precoding matrices and the associated constraints can be found in (Chen et al., 2007; Pan et al., 2004; Spencer et al., 2004).

\section{Problem statement}

To fully exploit multiuser diversity the following questions have to be addressed. In a spatial multiplexing opportunistic SDMA system with an excess number of SAs at the BS, how should the optimal set of spatially separable users be chosen? What is the appropriate allocation of the transmit/receive antennas (spatial beams) targeting the selected users? Since CCS pilots only provide a sub-optimal metric (i.e. CSI), how can a better metric be provided (i.e. instantaneous SINR) for such optimization problem while maintaining the same inherent feedback bandwidth and delay efficiency? For practical reasons such as cost and physical size, the number of SAs at the BS is greater than the number of OAs at the MS, as is the case 
in EUTRA. Algorithms that achieve spatial multiplexing gains such as V-BLAST receivers (Foschini, 1996), however, require that the number of antennas at the receiver is greater than or equal to the number of transmit antennas. Consequently, the number of DL spatial streams is limited by the number of OAs at the MS side. This situation results in a spatial DoF for the selection of the subset of transmit SA for per user DL transmission.

Note that according to the fourth assumption in section 2, it is assumed that the BD algorithm can only eliminate intracell CCI. However, the system is still limited by intercell CCI. Therefore, throughout this chapter, whenever the term interference is mentioned, it refers to CCI originated from transmitters in the adjacent cells.

\section{Interference-aware metric for downlink optimization}

In the following we make use of channel reciprocity which is best available in the TDD mode. The new interference-weighted feedback concept proposed in this chapter is applied to the UL CCS pilots. The first major contribution of this chapter is the use of modified pilots, termed UL IWCS pilots, which are proposed to replace the UL CCS pilots. In particular, the CCS pilots are modified to become IWCS pilots by weighting (dividing) the UL CCS pilots by the magnitude of the intercell CCI received at each MS. The UL IWCS pilots are then used at the BS to extract the CSI plus the level of the intercell CCI experienced by the respective MS. Thereby, the SINR at each active MS is conveyed to the respective BS without any additional signalling overhead. The key idea of applying the interference-weighting concept to the CCS pilots is depicted in Fig. 4 for a SISO case.

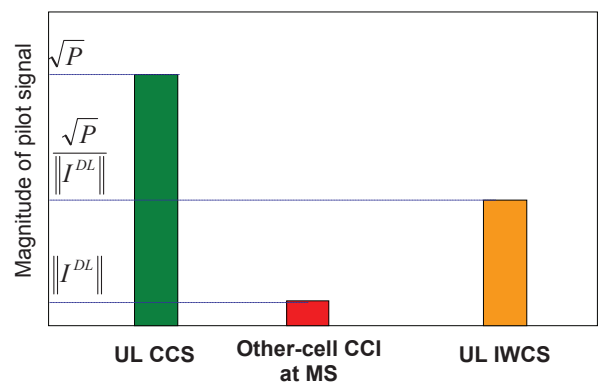

Fig. 4. Interference-weighting concept applied to the pilot signal in a SISO case

Consider an UL CCS transmission of a SDMA cellular interference-limited scenario with a BS equipped with $N_{\mathrm{BS}}$ SAs and $K$ active users, each equipped with $N_{\mathrm{MS}}$ OAs. A narrowband flat fading channel is assumed, i.e., a frequency subcarrier if orthogonal frequency division multiple access (OFDMA) or single carrier frequency division multiple access is used. We assume that both the BS and MSs experience sufficient local scattering. Therefore, both real and imaginary parts of the entries of $\mathbf{H}_{k}$ are samples of a zero-mean Gaussian distribution. Hence, the conventional channel sounding MS-to-BS pilots transmission can be modeled as follows:

$$
\mathbf{y}_{u}(t)=\mathbf{H}_{k}(t) \mathbf{z}_{k}(t)+\mathbf{n}_{k}(t)
$$

where $t$ is the time index. The predetermined pilot signal $\mathbf{z}_{k}(t)$ is a $N_{\mathrm{MS}}$-dimensional vector; the received signal $\mathbf{y}_{k}(t)$ is a $N_{\mathrm{BS}}$-dimensional vector. Conventional channel sounding pilots can be used to estimate two metrics: distance-dependent link gain (link budget), and the 
multipath fading channel coefficients (small-scale fading). By weighting (6) by the intercell CCI experienced by user $k$ in the downlink, the IWCS transmission can be written as follows:

$$
\mathbf{y}_{k}(t)=\frac{\mathbf{H}_{k}(t) \mathbf{z}_{k}(t)}{\left\|I_{k}^{\mathrm{DL}}(t-1)\right\|}+\mathbf{n}(t)
$$

where $\left\|I_{k}^{\mathrm{DL}}(t-1)\right\|$ is the amplitude of the intercell CCI experienced by $k^{\text {th }}$ MS at $(t-1)^{\text {th }}$ time interval. Consequently, the interference-weighting concept enables the sounding pilots to be used by the BS to obtain the DL interference-aware-metric $\mathbf{O}_{k}^{\mathrm{DL}-\mathrm{IAM}}(t) \in \mathbb{C}^{N_{\mathrm{BS}} \times N_{\mathrm{MS}}}$ which can be formulated as follows

$$
\mathbf{O}_{k}^{\mathrm{DL}-\mathrm{IAM}}(t)=\frac{\mathbf{H}_{k}(t)}{\left\|I_{k}^{\mathrm{DL}}(t-1)\right\|}
$$

From an information theoretic point of view, this metric (which can be considered as the square root of the instantaneous MIMO DL signal-to-interference ratio (SIR)) provides better feedback information compared to the CCS case. Consequently, the DL joint user scheduling and transmit SA selection can be improved since the DL interference-aware-metric can be used to obtain the SINR at the user side. Alternatively, quantized SINR can be fed back via the direct feedback method, but this requires transmission resources and longer time (potentially resulting in outdated feedback).

\subsection{Interference estimation}

From a practical point of view, the CCI experienced by a MS or a BS can be estimated by allowing the respective entity to sense the channel when no intended transmission is taking place. For instance, the CCI sensing period can be set for an active MS to be between the end of the DL period and the beginning of the switching time (DL to UL). Then, the MS can quantize the sensed CCI during the switching time period. Afterwards, a pilot weighted by the quantized magnitude of CCI can be transmitted during the subsequent UL period. This means that strong CCI will cause an 'artificial' attenuation of the pilot signal. This will pretend a bad channel at the BS, i.e the probability that the BS will schedule this particular resource block for this MS is reduced.

\subsection{Optimization methodology}

The general approach used in this chapter to maximize the sum capacity is a brute-force search. As in the example illustrated in Fig. 5, each BS, equipped with 4 SAs, will select 2 MSs each equipped with 2 OAs to access its spatial resources. Clearly, each MS experiences independent levels of CCI, and is subject to independent channel conditions on the desired link. For instance, the BS in cell 1 can schedule 2 users out of 3 possible candidates. For any active user, a BS can assign 2 out of 4 SAs to establish communication links. Using combinatorial basics, the BS has $\left(\begin{array}{l}3 \\ 2\end{array}\right)\left(\begin{array}{l}4 \\ 2\end{array}\right)=18$ options to select antennas and user pairs in the given example.

The procedure followed to extract the optimization metrics provided by IWCS pilots is illustrated in the example depicted in Fig. 6 which is based on the example of Fig. 5. Basically, each way in which the BS can distribute the 4 SAs among 2 out of 3 users forms a possible solution. From Fig. 6, two arbitrary solutions are highlighted by shading them with squares of different colors and different styles for the borderline. By considering the solution shaded by blue squares of solid borderline, it can be seen that MS1 is allocated the first two SAs, while 


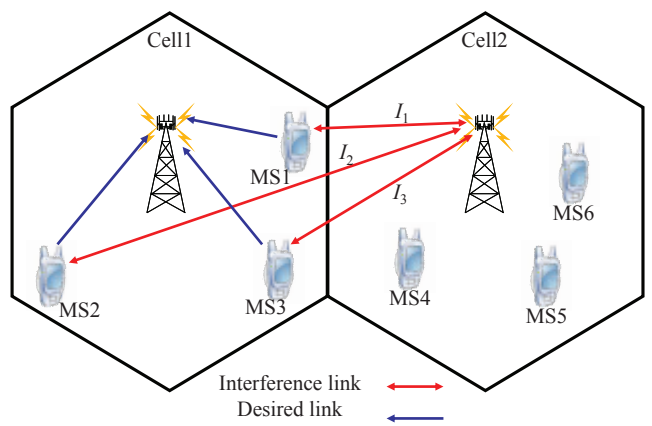

Fig. 5. Interference-limited multiuser MIMO system where each BS is equipped with 4 antennas, each MS is equipped with 2 antennas.

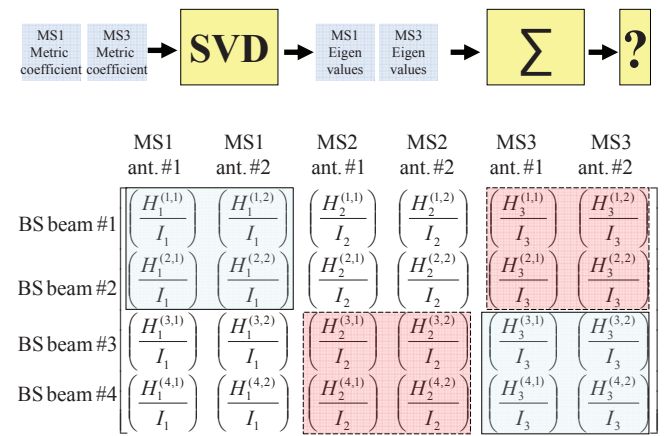

Fig. 6. Interference-limited virtual MU-MIMO matrix for the example illustrated in Fig. 5.

MS3 is allocated the last two antennas. Clearly, it can be seen that each SA allocation forms a $(2 \times 2)$ square matrix containing the coefficients of the optimization metric.

The next step is to obtain the eigenvalues of each square matrix via singular value decomposition (SVD). Afterwards, the eigenvalues of each group of selected users are summed. Finally, the summation of eigenvalues will be used to find the optimum solution among all possible solutions according to the different scheduling criteria. To examine all possible solutions, two approaches are considered: exhaustive search (ES) and HA. The details of the two approaches are discussed in section 8.

\section{Link-protection-aware metric for uplink and downlink optimization}

The main purpose of this section is to propose a method to accommodate the IWCS pilots to suit UL optimization. Since the amplitude of CCI experienced at the BS $\left\|I_{j}^{\mathrm{UL}}(t-1)\right\|$ (referred to as UL interference) where $\left(j \in 1, \ldots, N_{\mathrm{BS}}\right)$ is different from the CCI experienced at the MS $\left\|I_{k}^{\mathrm{DL}}(t-1)\right\|$, the DL interference-aware-metric in (8) is not suitable for UL optimization. In order to use the IWCS pilots for UL optimization, the BS weights each row of the metric defined in (8) by the received interference at the associated SA $\left\|I_{j}^{\mathrm{UL}}(t-1)\right\|$ at the BS, which is assumed to be known at the BS side. Thus, the new optimization metric, referred to as 
link-protection-aware-metric, $\mathbf{O}_{k}^{\operatorname{LPAM}}(t) \in \mathbb{C}^{1 \times N_{\mathrm{MS}}}$ can be formulated as follows:

$$
\mathbf{O}_{k}^{\mathrm{LPAM}}(t)=\frac{\mathbf{H}_{k}^{(j)}(t)}{\left\|I_{k}^{\mathrm{DL}}(t-1)\right\|\left\|I_{j}^{\mathrm{UL}}(t-1)\right\|}
$$

where $\mathbf{H}_{k}^{(j)}(t)$ is the $j^{\text {th }}$ row in $\mathbf{H}_{k}(t)$.

Basically, this metric allows the BS to decide which subset of receive antennas it should use for user $k$, and which users should be grouped together. Since the link-protection-aware-metric is inversely proportional to $\left\|I_{k}^{\mathrm{DL}}(t-1)\right\|$, a MS experiencing low interference level has higher chances to be selected. Due to channel reciprocity, a user that receives little interference from a set of users (in Tx mode) in a particular time slot, this user only causes little interference to the same set of users (now in Rx mode) in a different time slot. Therefore, the link-protection-aware-metric decreases the probability of scheduling users that are potential strong interferers. Consequently, this forms an inherent link-protection for the already established links in the neighboring cells. Similarly, this metric can be used for DL optimization to jointly select a subset of users experiencing favorable channel conditions and low intercell CCI to receive from a subset of SAs causing low intercell CCI to the neighboring cells. Note that the link-protection-aware-metric is simultaneously used to improve both UL and DL performance. Hence, the cross-layer scheduling for UL has to be the same as for DL, which reduces the scheduling time.

According to the MIMO literature (Foschini \& Gans, 1998; Telatar, 1999), if the channel matrix $\mathbf{H}_{k}$ is known at the BS, then the instantaneous DL MIMO channel capacity of user $k$ using fixed transmit power $\frac{P_{t}}{N_{\mathrm{BS}}}$ per antenna can be expressed as the sum of capacities of $r$ SISO channels each weighted with power gain $\lambda_{k_{i}}$ where $(i \in 1, \ldots, r), r$ is the rank of the channel, and $\lambda_{k_{i}}$ are the eigenvalues of $\mathbf{H}_{k} \mathbf{H}_{k}^{H} . P_{t}$ is the total transmit power at the BS. Assuming an interference-limited system, the instantaneous MIMO capacity of user $k$ can be expressed as follows:

$$
C_{k}=\sum_{i=1}^{r} \log _{2}\left(1+\frac{P_{t}}{N_{\mathrm{BS}} \times\left\|I_{k}^{\mathrm{DL}}\right\|^{2}} \lambda_{k_{i}}\right)
$$

By using the system model introduced above and (10), the primary objective is to find the optimum way, according to the scheduling criterion in use, in which the BS distribute $N_{\mathrm{BS}}$ antennas among $\frac{N_{\mathrm{BS}}}{N_{\mathrm{MS}}}$ spatially separable users out of user population of size $K$, where a selected user communicates with exactly $N_{\text {MS }}$ SAs at the BS. For instance, in the case of a maximum sum rate scheduling criterion, the optimum solution maximizes the capacity of the multiuser MIMO channel at the expense of fairness.

The sample-space population (SP) (the size of the pool containing all possible solutions) of such problem is formulated later in this chapter. The resulting optimization problem can be written as follows:

$$
C_{\max }=\underset{v \in \mathrm{SP}}{\arg \max } \sum_{k=1}^{\frac{N_{\mathrm{BS}}}{N_{\mathrm{MS}}}} C_{k}^{v}
$$

where SP are all possible choices of allocating the beams to the members of $v$, while $v$ represents a possible choice of grouping the scheduled users. 


\subsection{Summary of the optimization metrics}

In the DL, the following four metrics are used in conjunction with the different scheduling criteria (section IV summarizes them):

1 The scheduler only uses perfect instantaneous measurements of the MIMO multipath fading channel coefficients (ignoring the distance-dependent link-gain). This metric is fair by nature due to the uniform distribution of the users and the i.i.d. distributed fading, and it is referred to as DL blind-metric $\mathbf{O}_{k}^{\mathrm{DL}-\mathrm{BM}}(t)$.

2 The scheduler uses instantaneous measurements of the distance-dependent link-gain. In contrast, this metric is greedy by nature because it tends, in most of the cases, to select those users which are closer to the BS, and it is referred to as DL link-gain-aware-metric $\mathbf{O}_{k}^{\mathrm{DL}-\mathrm{LGAM}}(t)$.

Note, the first two metrics above are supported by the conventional channel sounding pilots and they do not provide the BS with information about interference at the mobile, $\left\|I_{k}^{\mathrm{DL}}(t-1)\right\|$.

3 The DL interference-aware-metric $\mathbf{O}_{k}^{\mathrm{DL}-\mathrm{IAM}}(t)$ defined in (8) is used. This metric is supported by the IWCS pilots or by means of explicit signalling which, however, requires additional resources for signalling traffic.

4 To foster link-protection awareness the metric developed in (9), $\mathbf{O}_{k}^{\mathrm{LPAM}}(t)$, is used.

Two cases are examined for the UL:

1 The scheduler uses instantaneous measurements of the CSI of each spatial stream (each row of $\mathbf{H}_{k}$ ) divided by the interference level experienced by the associated SA at the BS. This metric, referred as UL interference-aware-metric, $\mathbf{O}_{k}^{\mathrm{UL}-\mathrm{IAM}}(t) \in \mathbb{C}^{1 \times N_{\mathrm{MS}}}$ can be expressed as follows:

$$
\mathbf{O}_{k}^{\mathrm{UL}-\operatorname{IAM}}(t)=\frac{\mathbf{H}_{k}^{(j)}(t)}{\left\|I_{j}^{\mathrm{UL}}(t-1)\right\|}
$$

It is important to mention that this metric can be obtained using UL CCS pilots since UL interference $\left\|I_{j}^{\mathrm{UL}}(t-1)\right\|$ is available at the BS without feedback.

2 This is similar to the fourth case for the DL metrics which is defined as the link-protection-aware-metric in (9). This metric jointly considers UL and DL performance.

In summary, the results shown in section 10 are associated with four different metrics for DL transmission, and two different metrics for UL transmission.

\subsection{Numerical example}

To show the link-protection feature of the new metric defined in (9), a simple example is presented in Fig. 7. In this example, the arbitrary numbers quantifying the gain of each link and the interference experienced by each entity are used to estimate the achievable capacity using (10). It is assumed that cell 2 has an established DL transmission with MS3 and the argument for the achievable DL capacity is $\frac{\mathrm{H}_{\mathrm{MS} 3}}{\mathrm{I}_{\mathrm{MS} 3}}=\frac{9}{3}$. The neighboring BS, BS1, has got assigned the same time slot for UL transmission and it attempts to select between MS1 and MS2. If BS1 uses the UL interference-aware-metric, MS1 is scheduled for UL; $\frac{\mathrm{H}_{\mathrm{MS1}}}{\mathrm{I}_{\mathrm{BS} 1}}=\frac{6}{2}>$ $\frac{H_{\text {MS2 }}}{I_{\text {BS1 }}}=\frac{5}{2}$. Hence, the argument for the achievable UL capacity is $\frac{H_{M S 1}}{I_{B S 1}}=\frac{6}{2}$. As a result, it is assumed that the interference at MS3 increases to $\mathrm{I}_{\mathrm{MS} 3}=4.5$ due to the low shadowing 


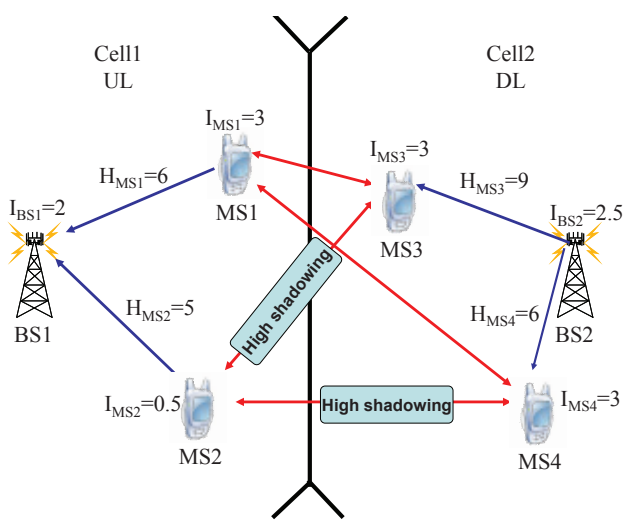

Fig. 7. Example of interference-limited 2-cell scenario with which the basic working principle of the link-protection-aware-metric is illustrated.

conditions between MS3 and MS1. This reduces the argument of the current achievable DL capacity in cell 2 to $\frac{9}{4.5}$. Thus, the arguments of the achievable system capacity are $\frac{6}{2}$ and $\frac{9}{4.5}$. In contrast, if BS1 uses the link-protection-aware-metric, MS2 is scheduled; $\frac{\mathrm{H}_{\mathrm{MS1}}}{\mathrm{I}_{\mathrm{MS} 1} \times \mathrm{I}_{\mathrm{BS} 1}}=$ $\frac{6}{3 \times 2}<\frac{\mathrm{H}_{\mathrm{MS2}}}{\mathrm{I}_{\mathrm{MS} 2} \times \mathrm{I}_{\mathrm{BS1}}}=\frac{5}{0.5 \times 2}$. Hence, the argument of the achievable UL capacity is $\frac{\mathrm{H}_{\mathrm{MS} 1}}{\mathrm{I}_{\mathrm{BS1}}}=\frac{5}{2}$. Consequently, the interference at MS3 does not change (due to the high shadowing between MS2 and MS3), and therefore, the arguments of the achievable cell capacity are $\frac{5}{2}$ and $\frac{9}{3}$ for cell 1 and cell 2, respectively. In summary, in the first case the sum of the arguments of the cell capacity is 5 whereas in the second case the sum is 5.5. Clearly, it can be seen from this example that the link-protection-aware-metric used in the second case improves the overall spectral efficiency.

\section{Heuristic algorithm for reduced computational complexity}

\subsection{Introduction}

Generally the ES is not practical due to its computational complexity. Therefore, in this section a heuristic algorithm is proposed to reduce the involved complexity.

\subsection{Exhaustive search mathematical model}

The complexity of exhaustive search approach for scheduling optimization of SDMA system depends on the total number of users $K$, the number of SAs at the BS $N_{\mathrm{BS}}$, and the number

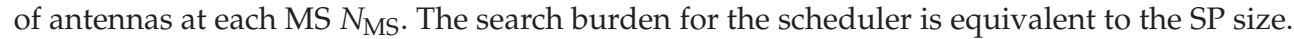
Using combinatorial fundamentals, (13) and (14) can be obtained. By comparing (13) with (14), it can be noticed that the multiuser diversity plays a significant role when $K>\frac{N_{\mathrm{BS}}}{N_{\mathrm{MS}}}$; due the fact that not all users can be scheduled.

If $K \leq \frac{N_{\mathrm{BS}}}{N_{\mathrm{MS}}}$

$$
\mathrm{SP}=\underbrace{\frac{N_{\mathrm{BS}} !}{\left(\left(N_{\mathrm{MS}} !\right)^{K}\left(N_{\mathrm{BS}}-K N_{\mathrm{MS}}\right) !\right)}}_{\text {SDMA DoF }} ;
$$


if $K>\frac{N_{\mathrm{BS}}}{N_{\mathrm{MS}}}$

$$
\mathrm{SP}=\underbrace{\frac{K !}{\left(\left(\frac{N_{\mathrm{BS}}}{N_{\mathrm{MS}}}\right) !\left(K-\frac{N_{\mathrm{BS}}}{N_{\mathrm{MS}}}\right) !\right)}}_{\text {Multiuser DoF }} \underbrace{\frac{N_{\mathrm{BS}} !}{\left(N_{\mathrm{MS}} !\right)\left(\frac{N_{\mathrm{BS}}}{N_{\mathrm{MS}}}\right)}}_{\text {SDMA DoF }} .
$$

It is important to mention that (13) and (14) are applicable only for scenarios in which $N_{\mathrm{BS}}$ is an integer multiple of $N_{\mathrm{MS}}$. To gain insight into the influence of each dimension of the DoF on the SP, Fig. 8, Fig. 9(a), and Fig. 9(b) are obtained assuming $N_{\mathrm{MS}}$ to be 2. By comparing

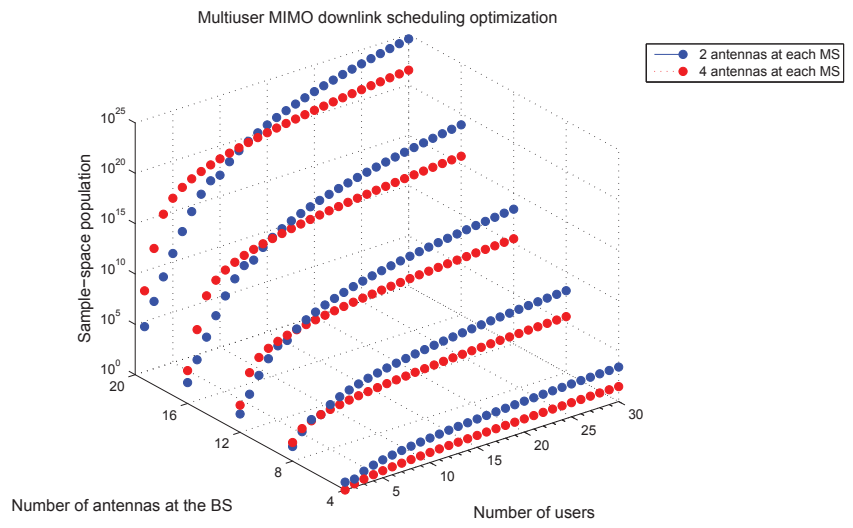

Fig. 8. The search complexity versus the number of SAs at the BS and the number of users, assuming 2 and 4 antennas at each MS

Fig. 9(a) with Fig. 9(b), it can be seen that SP grows polynomially with the number of users, and exponentially with the number of antennas at the BS (Learned et al., 1997). Clearly, the ES approach is computationally expensive for large number of users and antennas.

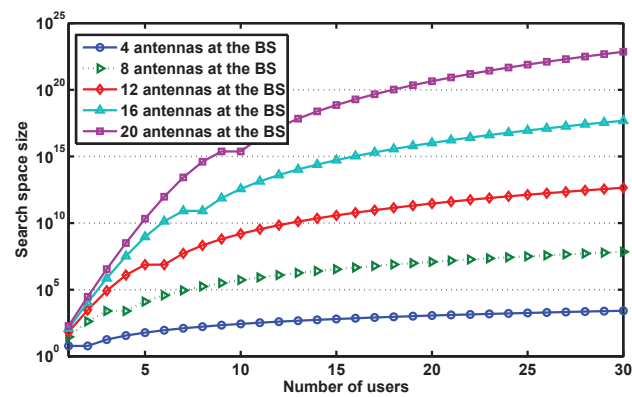

(a) Search space size versus the number of users

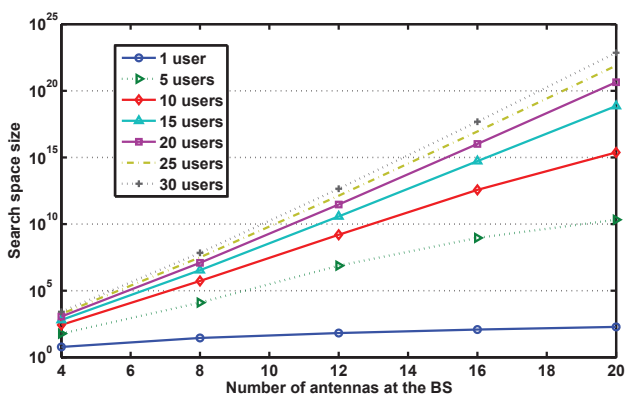

(b) Search size versus the number of SAs at the BS

Fig. 9. Search space size assuming 2 antennas at each MS 


\subsection{Heuristic to reduce complexity}

A HA is developed to significantly reduce the computational complexity of finding a close to optimum solution. The algorithm exploits two basic principles. The first one is based on the elimination of users that experience excessive interference while the second is based on an angle of arrival (AoA) sectorization approach. The former aims to reduce the SP by suppressing those users exposed to interference levels greater than a predetermined interference threshold. The latter converts the cell of interest (CoI) optimization problem into smaller optimization problems, which can be solved simultaneously. This can be done by sectorizing the cell based on the AoA of the uplink channel sounding signal. Subsequently, the antennas at the BS are distributed among these sectors according to the user density of each sector. As a consequence, the original SP of the CoI will be reduced significantly as the complexity is split into smaller parallel search spaces.

\subsection{Numerical examples}

To demonstrate the working mechanism of the HA, let us consider a cell with 28 users, each equipped with 4 antennas, and the BS equipped with 16 antennas. According to (14), the scheduler has to search through approximately $3.027 \times 10^{9}$ possibilities to find the optimum solution. By applying the interference based user elimination approach, let us assume that 8 users have been found to be exposed to severe interference levels. Similarly, assume that the cell can be equally divided into four sectors with the same user density. According to the proposed HA, the original optimization problem becomes four identical sector optimization problems. Hence, each sector has 4 associated antennas at the BS, and $\frac{28-8}{4}=5$ admissible users, each equipped with 4 antennas. Since only one user can be supported per sector, the scheduler has to search through 5 possibilities per sector, which can be done in parallel for all sectors.

To show the effect of the sectorization and the number of antennas at the MS, another example is used. In this example, a cell with 6 users, each equipped with 2 antennas, and the BS equipped with 12 antennas, is considered. Following a similar procedure as in the previous example, assume that all users are admissible, and that the cell can be equally sectorized into only 2 sectors with the same user density. Thus, each sector has 6 associated antennas at the BS, and 3 users, each equipped with 2 antennas. According to (13), the optimization problem with a search space of $3.992 \times 10^{7}$ is converted into 2 identical optimization problems, each with a search space of 120 . It is worth noting in this example that multiuser diversity has not been exploited, and a simple sectorization technique is applied. However, the scheduler search burden is significantly reduced, and thus the practicability of the proposed interference-aware antenna selection and scheduling algorithm is greatly enhanced.

\section{Scheduling criteria}

Scheduling criteria can be generally classified into two groups: greedy and fair. In this chapter, the considered optimization metrics are tested using three scheduling criteria. Specifically, a greedy criterion referred to as maximum capacity (MC) (Borst \& Whiting, 2003; Gesbert et al., 2007), and two fair criteria referred to as proportional-fair (PF) (Chaponniere et al., 2002; Viswanath et al., 2002) and score-based (SB) (Bonald, 2004) are considered. 


\subsection{Maximum capacity scheduling criterion}

MC criteria always assigns the antennas to those users with the highest eigenvalue sum, hence, it is efficient in terms of overall throughput but may look oppressive for low SINR users, typically located far from the BS (cell-edge users).

The achievable capacity according to MC scheduling criterion can be formulated as follows:

$$
C^{\mathrm{MC}}=\underset{v \subset \mathrm{SP}}{\arg \max }\left\{C_{v}\right\},
$$

where $C^{\mathrm{MC}}$ is the achievable capacity using $\mathrm{MC}$ criterion, $C_{v}$ is the instantaneous capacity of the $v^{\text {th }}$ option.

\subsection{Proportional fair scheduling criterion}

The PF criterion seeks to assign the antennas to those users with the highest eigenvalue sum relative to their mean eigenvalue sum. This causes the scheduler to realize a reasonable trade-off between throughput efficiency and fairness.

The achievable capacity according to PF scheduling criterion can be formulated as follows:

$$
C^{\mathrm{PF}}=\underset{v \subset \mathrm{SP}}{\arg \max }\left\{\frac{C_{v}}{\overline{\bar{C}}_{v}}\right\},
$$

where $C^{\mathrm{PF}}$ is the achievable capacity using PF criterion, $C_{v}$ and $\bar{C}_{v}$ are the instantaneous and average capacity of the $v^{\text {th }}$ option, respectively.

\subsection{Score-based scheduling criterion}

The SB scheduler assigns the antennas to those users with the best scores. The score corresponds to the rank of the current eigenvalue sum among the past values observed over a time window of a particular length, $W$ (Bonald, 2004).

The achievable capacity according to SB scheduling criterion can be formulated as follows:

$$
\mathrm{C}^{\mathrm{SB}}=\underset{v \subset \mathrm{SP}}{\arg \min }\left\{S_{v}\right\}
$$

where $C^{S B}$ is the achievable capacity using the $\mathrm{SB}$ criterion, and $S_{v}$ is the score of the instantaneous capacity of the $v^{\text {th }}$ option among the past values observed over the time window $W$. The throughput-fairness trade-off is customizable by changing the window size $W$. More specifically, setting the window size equal to one, this will result in a similar behavior as observed with the MC criterion. A large window size converges to the behavior of the PF scheduler.

Since the score, $S_{v}$, is an integer number several scheduling scenarios could result in the same best rank (the first position). In this case, the solution which achieves the highest throughput is selected. Therefore, in this chapter the SB criterion effectively results in a hybrid version of both the SB scheduler and the MC scheduler.

\section{Results and discussion}

In this section, the performance of the different optimization metrics is assessed using the cumulative distribution function (cdf) of per-user capacity and cell capacity for both UL and DL. The per-user capacity in this work is the $2 \times 2$ spatial multiplexing MIMO capacity which 
can be calculated using (10). The cell capacity is the sum capacity of the group of scheduled users. In order to shed some light on fairness of the considered optimization metrics, the cdf of the scheduled (served) user distance to the BS is obtained for all considered scheduling criteria.

A two-tier cellular platform consisting of hexagonal cells is used in the simulation. In each cell, the BS and MSs are equipped with (6 or 8) and 2 antennas, respectively. Using the parameters given in Table 1, the 19-cell cellular TDD system with uniform user distribution, as shown in Fig. 10, is simulated using the Monte Carlo method. The channel matrix of $k^{\text {th }}$ user $\mathbf{H}_{k}$ is a zero-mean i.i.d. complex Gaussian random matrix. The system level performance evaluation is based on both central cell and wrap-around techniques. The DL and UL performances are

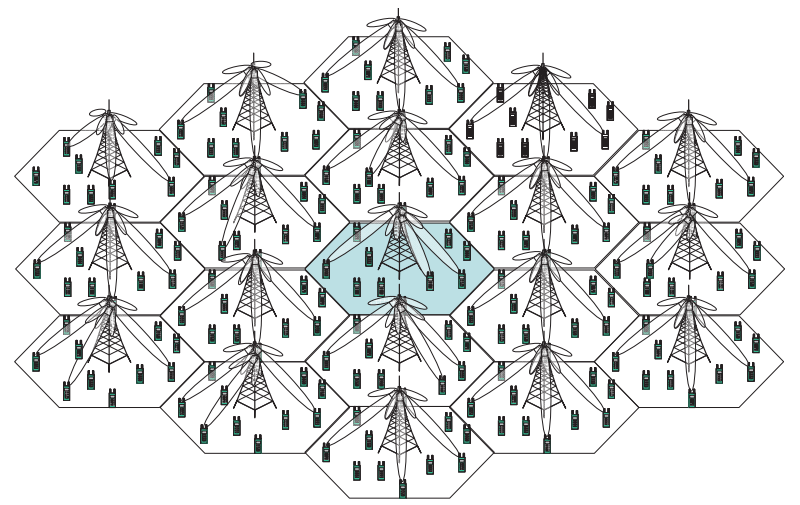

Fig. 10. Interference-limited multiuser MIMO system implementing orthogonal SDMA

analyzed for asynchronous UL/DL transmissions, where the two possible link-directions (UL or DL) occur with equal probability and independently from each other. All entities (BSs and MSs) in the system use fixed transmit power of $30 \mathrm{dBm}$.

In the wrap-around technique, the multicell layout is folded such that cells on the right side of the network are connected with cells on the left side and, similarly, cells in the upper part of the network get connected to cells in the lower part. The created area may be seen as borderless, but with a finite surface, and it may be visualized as a torus. The wrap-around technique is suitable for both downlink and uplink simulations. One of the main advantages of wrap-around technique is that the decision taken by a scheduler in a particular cell influences the scheduling behavior in the adjacent cells. Such an observation cannot be collected using the central cell technique. Another advantage compared to the central cell technique is that simulation data can be collected from all cells, which may reduce the required simulation time to collect sufficient statistics.

\subsection{Downlink performance with interference awareness}

In order to avoid a huge SP size, the BS is assumed to be equipped with 6 SAs. Therefore, the same time-frequency resource is simultaneously used, at maximum, three times within the same cell for three different users. The results in Fig. 11(a) show that the cell capacity can be significantly enhanced if knowledge of interference is taken into account in the antenna selection and MIMO user scheduling process. For example it can be seen from Fig. 11(a), for the MC criterion (unmarked curves), that the median of the DL capacity for the 


\begin{tabular}{|l|c|}
\hline $\begin{array}{l}\text { Channel propagation } \\
\text { environment }\end{array}$ & Suburban micro cell \\
\hline BS-to-BS distance & $300-500 \mathrm{~m}$ \\
\hline Number of interfering tiers & 2 \\
\hline Pilot Tx power & $30 \mathrm{dBm}$ \\
\hline Number of users & $2 \mathrm{GHz}$ \\
\hline Carrier frequency & $35 \mathrm{~m}$ \\
\hline $\begin{array}{l}\text { Minimum distance between } \\
\text { MS and BS }\end{array}$ & $6-8$ \\
\hline Number of antennas at BS & 2 \\
\hline Number of antennas at MS & $8 \mathrm{~dB}$ \\
\hline $\begin{array}{l}\text { log-normal shadowing } \\
\text { standard deviation, } \sigma_{\mathrm{S}}\end{array}$ & $31.5+35 \log 10(d)$ \\
\hline $\begin{array}{l}\text { Correlation distance } \\
\text { of shadowing }\end{array}$ & \\
\hline Path loss model $(\mathrm{dB})$ & $\mathrm{m}(\mathrm{as}$ in UMTS 30.03$)$ \\
\hline
\end{tabular}

Table 1. Simulation parameters

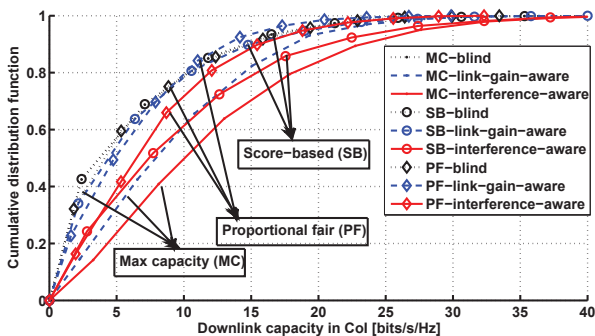

(a) Cell performance

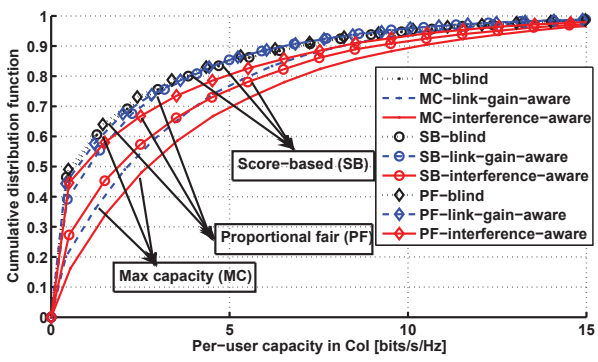

(b) User performance

Fig. 11. Downlink performance comparison among the DL interference-aware and both blind and link-gain-aware metrics using ES approach

DL interference-aware-metric (using the novel concept of interference-weighted pilots) is 10 bps $/ \mathrm{Hz}$ while it is 7.5 and $4 \mathrm{bps} / \mathrm{Hz}$ for the link-gain-aware-metric and the blind-metric, respectively. As expected from the greedy characteristics of the link-gain-aware-metric, it can be seen that it outperforms the blind-metric. The greediness of these metrics will be revisited later when the issue of fairness is discussed. Similarly, the per-user capacity in the case of the DL interference-aware-metric is substantially improved for all scheduling criteria. From Fig. 11(b), for the SB criterion (circle-marked curves), it can be seen that the median of the per-user capacity resulting from the DL interference-aware-metric is $2 \mathrm{bps} / \mathrm{Hz}$ while it is 1 and 0.5 bps/Hz for link-gain-aware-metric and the blind-metric, respectively.

The results in Fig. 12 are obtained for the case of a cell radius of $300 \mathrm{~m}$. It is shown that both DL interference-aware-metric and link-gain-aware-metric prioritize the users closer to the BS when the MC criterion is used. For instance, Fig. 12, assuming the MC criterion (unmarked curves), shows that the median of the served user distance to the 
BS of the DL interference-aware-metric and the link-gain-aware-metric are $160 \mathrm{~m}$ and $140 \mathrm{~m}$, respectively, while it is $190 \mathrm{~m}$ for the blind-metric case. Alternatively, when the PF criterion (diamond-marked curves) is used the DL interference-aware-metric shows high level of fairness (median is $220 \mathrm{~m}$ ) compared to both blind-metric (median is $175 \mathrm{~m}$ ) and link-gain-aware-metric (median is $210 \mathrm{~m}$ ).

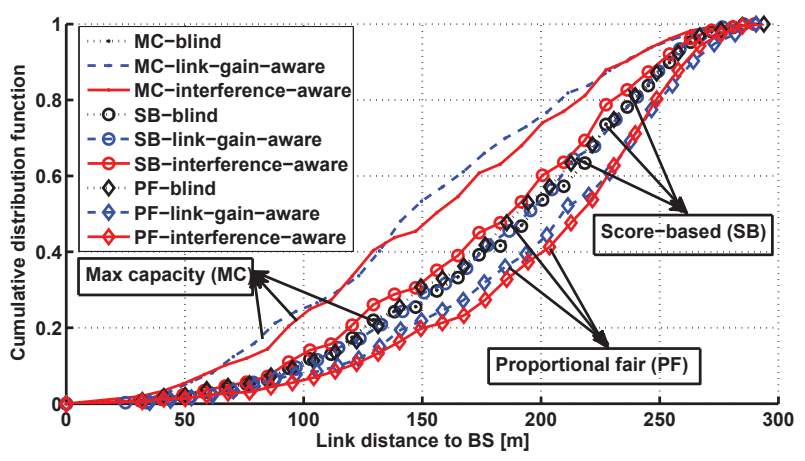

Fig. 12. Fairness comparison among the three considered scheduling criteria in terms of the distance between the BS and the scheduled user.

It is worth noting that the distance distribution of the served users associated with the blind-metric is only slightly affected by using the SB and the PF criteria. This is expected due to the inherent fairness of this metric which is a consequence of the i.i.d. distributed small-scale fading and the random distribution of the users.

\subsection{Performance of heuristic algorithm}

In order to study the efficiency of the HA, a DL scenario is simulated under the following assumptions: 8 SAs at the BS, 2 antennas at each MS, fixed sectorization is used to equally divide the cell into two sectors, only the best 8 users in terms of the experienced intercell CCI are considered in each cell, and the MC criterion is used. It has been found using simulations that the search space has been reduced from 176400 to 80 on average (depending on the user density per sector). This is achieved at the following cost: The median of the achievable throughut using DL interference-aware-metric in combination with the HA approach only reaches approximately $90 \%$ of the median of the optimum capacity using the exhaustive search approach. This can be seen in Fig. 13.

Since the ES approach is computationally too expensive, simulation results for larger SP sizes cannot be obtained within reasonable time. Therefore, all the subsequent results are obtained using the HA approach due to its low complexity and close to optimum performance as demonstrated in Fig. 13. Furthermore, in order to increase the spatial SDMA DoF, the BS is assumed to be equipped with 8 SAs, hence 4 users can be scheduled.

\subsection{Uplink performance with link-protection awareness}

From Fig. 14(a), for the MC criterion (square-marked curves), it can be seen that the UL median cell capacity using the link-protection-aware-metric, (9), is $27 \mathrm{bps} / \mathrm{Hz}$ which corresponds to an enhancement of $12.5 \%$ of the UL median cell capacity using the UL interference-aware-metric, (12), which is $24 \mathrm{bps} / \mathrm{Hz}$. 


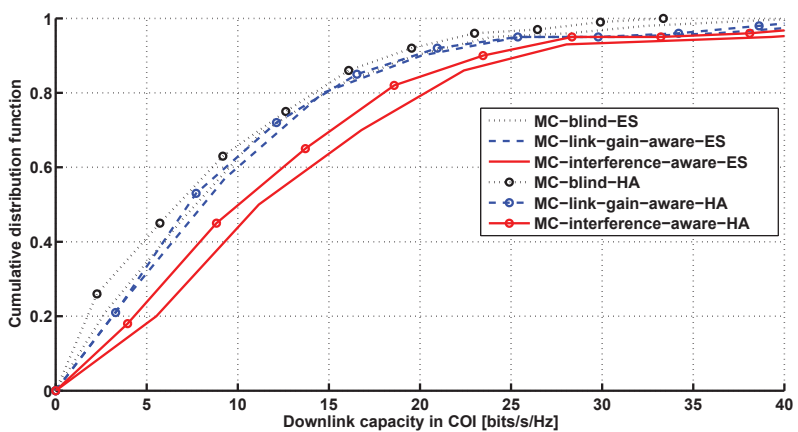

Fig. 13. Throughput comparison for the metrics considered in the previous section for the MC criterion using both ES and HA approaches

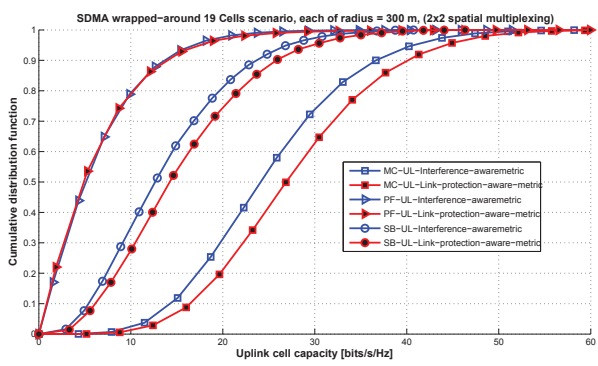

(a) Cell performance

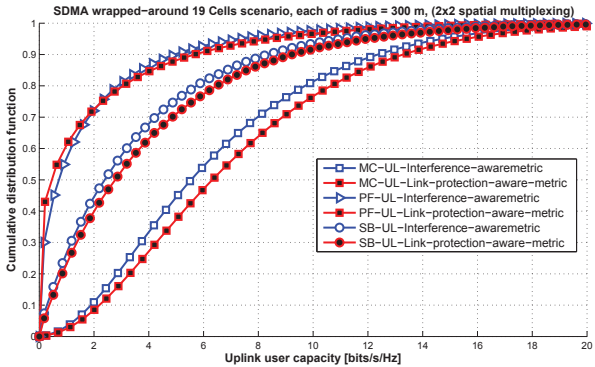

(b) User performance

Fig. 14. Uplink performance comparison between the UL interference-aware-metric and the link-protection-aware-metric

Notice that the link-protection-aware-metric, (9), jointly considers UL and DL and it implicitly provides the BS with CCI interference observed at the MSs. Since channel reciprocity is assumed, a user that observes high interference in the DL will in turn cause high interference to the corresponding users in the other cell when they are receiving data from their BS. Therefore, not scheduling a user that observes high interference is beneficial in two ways: (a) other users might observe less interference at these particular transmission resources, and scheduling these users would result in higher user capacity or reduced transmission power; and (b) interference caused to other users in the neighboring cells is automatically kept a low level. This effectively results in interference-aware radio resource management. These observations are affirmed by noting the results of the per-user capacity of the link-protection-aware-metric, which outperforms the UL interference-aware-metric for all scheduling criteria considered. For instance, from Fig. 14(b), for the SB criterion (circle-marked curves), it can be seen that the median of the per-user capacity resulting from the link-protection-aware-metric is $2.9 \mathrm{bps} / \mathrm{Hz}$ while it is $2.5 \mathrm{bps} / \mathrm{Hz}$ for the UL interference-aware-metric. However, the PF criterion (triangle-marked curves) does not provide a noticeable improvement due to the inherent fairness. The fairness constraint prioritizes a fair allocation of the spatial resources among the users over preventing strong interferers from being scheduled. 


\subsection{Downlink performance with link-protection awareness}

The same analysis carried out in the previous subsection for the UL is now applied to the DL. From Fig. 15(a), for the MC criterion (square-marked curves), it can be seen that the median

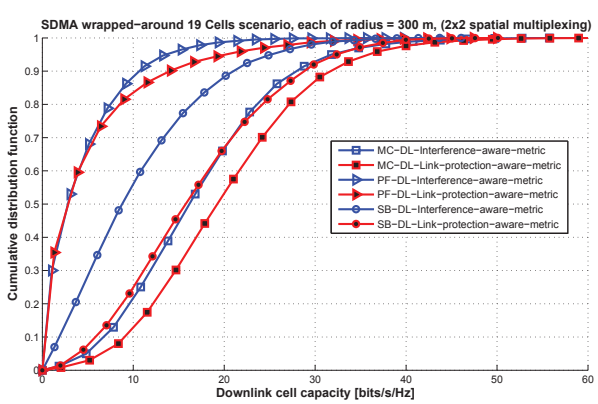

(a) Cell performance

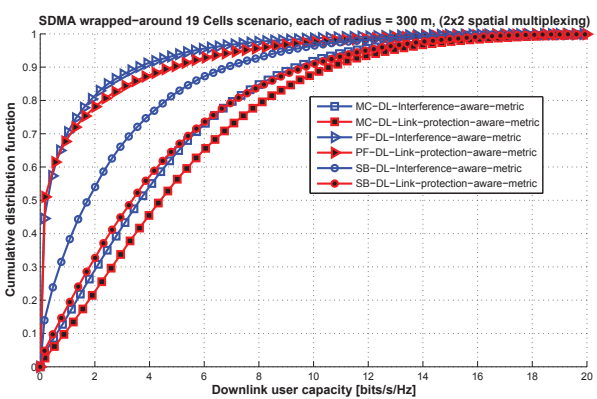

(b) User performance

Fig. 15. Downlink performance comparison between the DL interference-aware-metric and the link-protection-aware-metric.

of the DL capacity using the link-protection-aware-metric, (9), is $19 \mathrm{bps} / \mathrm{Hz}$ while it is 15 bps/Hz for the DL interference-aware-metric, (8). Similarly, the per-user capacity in the case of the link-protection-aware-metric is improved for all scheduling criteria. From Fig. 15(b), for the SB criterion (circle-marked curves), it can be seen that the median of the per-user capacity resulting from the link-protection-aware-metric is $3.5 \mathrm{bps} / \mathrm{Hz}$ while it is $1.9 \mathrm{bps} / \mathrm{Hz}$ for the DL interference-aware-metric.

Irrespective of the nature of the employed scheduling criterion, the results show that the gain in the UL is lower compared to the DL. This primarily is due to the fact that BS-to-BS interference degrades the link-protection selectivity in UL. Given that all antenna groups at the BS site are in close proximity, the large-scale fading (log-normal shadowing) on the interference links can, thus, be considered the same for this case. Therefore, the variations among the interference links depend only on the small-scale fading.

On the other hand, the tendency of greediness of the SB criterion, explained in section 9, allows for the exploitation of multiuser diversity in the DL given the high user density. Furthermore, due to the relatively high variance of the statistics of DL interference, a marginal improvement is achieved using the PF criterion, as it can be seen in Fig. 15. The gains are only materialized at the high interference percentiles due to the emphasis on fairness when using the PF criterion (triangle-marked curves).

\section{Conclusions}

Weighting the UL channel sounding pilots by the instantaneous CCI observed locally (referred to as interference-weighted channel sounding pilots) enables the users to implicitly convey the level of CCI to its BS along with channel state information. In addition, ICIC is achieved without any explicit inter-cell signalling as follows: A user that experiences high CCI in the DL would equally cause high interference to the same users that caused this interference when the transmission directions are reversed (due to channel reciprocity). The pilot-weighting mechanism "artificially" deteriorates the channel in this case so that this particular user will most probably be scheduled on a different channel which is beneficial for this user as well 
as for the interfering users in the neighboring cells (resulting in interference coordination). If additionally the modified pilots are weighted by the UL interference (observed at the BS), this provides combined knowledge of the interference at both ends. It has been shown that this additional information, for example, enables interference-aware user scheduling to improve the capacity compared to systems which only utilize the conventional channel sounding pilots.

It has been found that compared to both blind-metric and link-gain-aware-metric, a capacity gain of $150 \%$ and $35 \%$, respectively, at the $10^{\text {th }}$ percentile can be achieved when the novel downlink interference-aware-metric is used assuming the maximum capacity criterion. By considering the score-based policy, simulations show that using the link-protection-aware-metric results in a capacity gain of $230 \%$ and $15 \%$ at the $10^{\text {th }}$ percentile compared to both downlink and uplink interference-aware-metric, respectively. Marginal capacity gains have been obtained for the PF policy which ensures fairness at the expense of capacity efficiency. However, please notice that for the sake of conciseness only a single channel has bee assumed in this study. Higher gains are envisaged for the PF policy if a broadband OFDMA system with multiple resource blocks would have been considered.

Utilizing the proposed heuristic algorithm significantly reduces the computational complexity to approximately $0.05 \%$ of the complexity of the exhaustive search approach. This reduction in complexity is achieved at the cost of $8 \%$ loss at the $10^{\text {th }}$ percentile cell capacity.

\section{References}

Abe, T. \& Bauch, G. (2007). Differential codebook mimo precoding technique, Global Telecommunications Conference, 2007. GLOBECOM '07. IEEE, pp. 3963 -3968.

Abualhiga, R. \& Haas, H. (2008). Implicit Pilot-Borne Interference Feedback for Multiuser MIMO TDD Systems, Proc. of the International Symposium on Spread Spectrum Techniques and Applications (ISSSTA), IEEE, Bologna, Italy, pp. 334-338.

Airy, M., Bhadra, S., Heath, R. \& Shakkottai, S. (2006). Transmit Precoding for the Multiple Antenna Broadcast Channel, Proc. of the 63rd Vehicular Technology Conference (VTC 06), Vol. 3, IEEE, Melbourne, Australia, pp. 1396-1400.

Ali, S. H., Lee, K.-D. \& Leung, V. C. M. (2007). Dynamic Resource Allocation in OFDMA Wireless Metropolitan Area Networks, IEEE Wireless Communications 14(1): 6-13.

Bahceci, I., Duman, T. \& Altunbasak, Y. (2003). Antenna Selection for Multiple-Antenna Transmission Systems: Performance Analysis and Code Construction, IEEE Transactions on Information Theory 49(10): 2669-2681.

Bauch, G. \& Dietl, G. (2008a). Enhanced mimo for imt-advanced wireless systems, 2008 IET Seminar on Wideband and Ultrawideband Systems and Technologies: Evaluating current Research and Development, pp. $1-21$.

Bauch, G. \& Dietl, G. (2008b). Multi-user mimo for achieving imt-advanced requirements, International Conference on Telecommunications (ICT 2008), pp. 1-7.

Blum, R. (2003). MIMO Capacity with Interference, IEEE Journal on Selected Areas in Communications 21(5): 793-801.

Bonald, T. (2004). A Score-Based Opportunistic Scheduler for Fading Radio Channels, Proc. of the European Wireless Conference (EWC), Barcelona, Spain.

Borst, S. \& Whiting, P. (2003). Dynamic channel-sensitive scheduling algorithms for wireless data throughput optimization, IEEE Transactions on Vehicular Technology 52(3): 569-586. 
Catreux, S., Driessen, P. \& Greenstein, L. (2002). Data Throughputs Using Multiple-Input Multiple-Output (MIMO) Techniques in a Noise-Limited Cellular Environment, IEEE Transactions on Wireless Communications 1(2): 226-235.

Chae, C.-B., Mazzarese, D. \& Heath, R. W. (2006). Coordinated Beamforming for Multiuser MIMO Systems with Limited Feedforward, Fortieth Asilomar Conference on Signals, Systems and Computers (ACSSC) pp. 1511-1515.

Chaponniere, E. F. Black, P. J., Holtzman, J. M. \& Tse, D. N. C. (2002). Transmitter Directed Code Division Multiple Access System Using Path Diversity to Equitably Maximize Throughput, US Patent 6449490 .

Chen, R., Heath, R. W. \& Andrews, J. G. (2007). Transmit Selection Diversity for Unitary Precoded Multiuser Spatial Multiplexing Systems With Linear Receivers, IEEE Transactions on Signal Processing 55(3): 1159-1171.

Choi, L.-U. \& Murch, R. (2004). A Transmit Preprocessing Technique for Multiuser MIMO Systems Using a Decomposition Approach, IEEE Transactions on Wireless Communications 3(1): 20-24.

Choi, W., Forenza, A., Andrews, J. G. \& Heath Jr., R. W. (2006). Capacity of Opportunistic Space Division Multiple Access with Beam Selection, Proc. of the Global Telecommunications Conference (GLOBECOM 06), IEEE, San Francisco, USA, pp. 1-5.

Chung, S. T., Lozano, A. \& Huang, H. (2001a). Approaching Eigenmode BLAST Channel Capacity Using V-BLAST With Rate and Power Feedback, Proc. of the 54th Vehicular Technology Conference (VTC 01), Vol. 2, Atlantic City, New Jersey, pp. 915-919.

Chung, S. T., Lozano, A. \& Huang, H. (2001b). Low Complexity Algorithm for Rate and Power Quantization in Extended V-BLAST, Proc. of the 2001 IEEE 53rd Vehicular Technology Conference, Vol. 2, Atlantic City, New Jersey, pp. 910-914.

Costa, M. (1983). Writing on Dirty Paper, IEEE Transactions on Information Theory 29(3): 439-441.

Dai, H., Molisch, A. \& Poor, V. H. (2004). Downlink Capacity of Interference-Limited MIMO Systems with Joint Detection, IEEE Transactions on Wireless Communications 3(2): 442-453.

Foschini, G. J. (1996). Layered Space-Time Architecture for Wireless Communication in a Fading Environment when Using Multi-Element Antennas, Bell Labs Technical Journal 1(2): 41-59.

Foschini, G. J. \& Gans, M. J. (1998). On Limits of Wireless Communications in a Fading Environment when Using Multiple Antennas, Wireless Personal Communications 6(6): 311-335.

Fragouli, C., Al-Dhahir, N. \& Turin, W. (2003). Training-Based Channel Estimation for Multiple-Antenna Broadband Transmissions, IEEE Transactions on Wireless Communications 2(2): 384-391.

Fuchs, M. \& Del Galdo, G. \& Haardt, M. (2007). Low-Complexity Space-Time-Frequency Scheduling for MIMO Systems With SDMA, IEEE Transactions on Vehicular Technology 56(5): 2775-2784.

Gallen, C. (2009). In 2014 Monthly Mobile Data Traffic Will Exceed 2008 Total, ABI Research, Retrieved January 3, 2011, from www.abiresearch.com/press/

Gesbert, D., Kiani, S. G., Gjendemsjø, A. \& Øien, G. E. (2007). Adaptation, Coordination, and Distributed Resource Allocation in Interference-Limited Wireless Networks, Proc. of the 7th IEEE International Symposium on Wireless Communication Systems 95(12): 2393-2409. 
Ghrayeb, A. \& Duman, T. (2002). Performance analysis of MIMO Systems with Antenna Selection Over Quasi-Static Fading Channels, pp. 333-337.

Goldsmith, A., Jafar, S., Jindal, N. \& Vishwanath, S. (2003). Capacity Limits of MIMO Channels, IEEE Journal on Selected Areas in Communication 21(5): 684-702.

Gore, D., Heath, R. \& Paulraj, A. (2002). Statistical Antenna Selection for Spatial Multiplexing Systems, Proc. of the International Conference on Communications (ICC 02), Vol. 1, New York, USA, pp. 450-454.

Gore, D. \& Paulraj, A. (2002). MIMO Antenna Subset Selection with Space-Time Coding, IEEE Transactions on Signal Processing 50(10): 2580-2588.

Gorokhov, A., Gore, D. \& Paulraj, A. (2003). Receive Antenna Selection for MIMO Flat-Fading Channels: Theory and Algorithms, IEEE Transactions on Information Theory 49(10): 2687-2696.

Haas, H. \& McLaughlin, S. (eds) (2008). Next Generation Mobile Access Technologies: Implementing TDD, Cambridge University Press, ISBN: 13:9780521826228.

Hassibi, B. \& Hochwald, B. M. (2003). How Much Training is Needed in Multiple-Antenna Wireless Links?, IEEE Transactions on Information Theory 49: 951-963.

Heath, R. \& Paulraj, A. (2001). Antenna Selection for Spatial Multiplexing Systems Based on Minimum Error Rate, Proc. of the International Conference on Communications (ICC 01), Vol. 7, Helsinki, Finland, pp. 2276-2280.

Hochwald, B., Peel, C. \& Swindlehurst, A. (2005). A Vector-Perturbation Technique for Near-Capacity Multiantenna Multiuser Communication. part II: Perturbation, IEEE Transactions on Communications 53(3): 537-544.

Koutsimanis, C. \& Fodor, G. (2008). A Dynamic Resource Allocation Scheme for Guaranteed Bit Rate Services in OFDMA Networks, Proc. of the IEEE International Conference on Communications (ICC 08), pp. $2524-2530$.

Kusume, K., Joham, M., Utschick, W. \& Bauch, G. (2007). Cholesky factorization with symmetric permutation applied to detecting and precoding spatially multiplexed data streams, IEEE Transactions on Signal Processing 55(6): 3089 -3103.

Learned, R., Willsky, A. \& Boroson, D. (1997). Low complexity optimal joint detection for oversaturated multiple access communications, IEEE Transactions on Signal Processing 45(1): 113-123.

Love, D. J. \& Heath, R. (2005). Limited Feedback Unitary Precoding for Spatial Multiplexing Systems, IEEE Transactions on Information Theory 51(8): 2967-2976.

Love, D. J., Heath, R. \& Strohmer, T. (2003). Grassmannian Beamforming for Multiple-Input Multiple-Output Wireless Systems, Proc. of the International Conference on Communications (ICC 03), Vol. 4, IEEE, pp. 2618-2622.

Love, D. J., Heath, R., Santipach, W. \& Honig, M. L. (2004). What is the Value of Limited Feedback for MIMO Channels, IEEE Communications Magazine .

Molisch, A., Win, M. \& Winters, J. (2001). Capacity of MIMO Systems with Antenna Selection, Proc. of the International Conference on Communications (ICC 01), Vol. 2, pp. 570-574.

Molisch, A., Win, M. \& Winters, J. (2003). Reduced-Complexity Transmit/Receive-Diversity Systems, IEEE Transactions on Signal Processing 51(11): 2729-2738.

Mukkavilli, K., Sabharwal, A., Aazhang, B. \& Erkip, E. (2002). Performance Limits on Beamforming with Finite Rate Feedback for Multiple Antenna Systems, Proc. of the 36th Asilomar Conference on Signals, Systems and Computers, Vol. 1, pp. 536-540. 
Mukkavilli, K., Sabharwal, A., Erkip, E. \& Aazhang, B. (2003). On Beamforming with Finite Rate Feedback in Multiple-Antenna Systems, IEEE Transactions on Information Theory 49(10): 2562-2579.

Pan, Z., Wong, K.-K. \& Ng, T.-S. (2004). Generalized Multiuser Orthogonal Space-Division Multiplexing, IEEE Transactions on Wireless Communications 3(6): 1969-1973.

Popovic, B. (1992). Generalized chirp-like polyphase sequences with optimal correlation properties, IEEE Transactions on Information Theory 38: 1406-1409.

Schubert, M. \& Boche, H. (2004). Solution of the Multiuser Downlink Beamforming Problem with Individual SINR Constraints, IEEE Transactions on Vehicular Technology 53(1): 18-28.

Seidel, E. (2008). Progress on "LTE Advanced" - the New 4G Standard, Newsletter, NOMOR, Munich, Germany.

Sesia, S., Toufik, I. \& Baker, M. (2009). LTE - The UMTS Long Term Evolution: From Theory to Practice, Wiley.

Shen, Z., Chen, R., Andrews, J., Heath, R. \& Evans, B. (2005). Low Complexity User Selection Algorithms for Multiuser MIMO Systems with Block Diagonalization, Proc. of the 39th Asilomar Conference on Signals, Systems and Computers., pp. 628-632.

Shi, S., Schubert, M. \& Boche, H. (2008). Downlink MMSE Transceiver Optimization for Multiuser MIMO Systems: MMSE Balancing, IEEE Transactions on Signal Processing 56(8): 3702-3712.

Spencer, Q., Swindlehurst, A. \& Haardt, M. (2004). Zero-Forcing Methods for Downlink Spatial Multiplexing in Multiuser MIMO Channels, IEEE Transactions on Signal Processing 52(2): 461-471.

Telatar, E. (1999). Capacity of Multi-Antenna Gaussian Channels, European Transaction on Telecommunication 10(6): 585-595.

Vishwanath, S., Jindal, N. \& Goldsmith, A. (2003). Duality, Achievable Rates, and Sum-Rate Capacity of Gaussian MIMO Broadcast Channels, IEEE Transactions on Information Theory 49(10): 2658-2668.

Viswanath, P., Tse, D. \& Laroia, R. (2002). Opportunistic Beamforming Using Dumb Antennas, Proc. of the International Symposium on Information Theory, IEEE, p. 449.

Wang, C. \& Murch, R. (2005). Adaptive Cross-Layer Resource Allocation for Downlink Multi-User MIMO Wireless System, Proc. of the 61st Vehicular Technology Conference (VTC 05), Vol. 3, pp. 1628-1632.

Weingarten, H., Steinberg, Y. \& Shamai, S. (2004). The Capacity Region of the Gaussian MIMO Broadcast Channel, Proc. of the International Symposium on Information Theory (ISIT 04), Chicago, USA, pp. 174-182.

Windpassinger, C., Fischer, R., Vencel, T. \& Huber, J. (2004). Precoding in Multiantenna and Multiuser Communications, IEEE Transactions on Wireless Communications 3(4): 1305-1316.

Wong, K.-K., Murch, R. \& Letaief, K. (2003). A Joint-Channel Diagonalization for Multiuser MIMO Antenna Systems, IEEE Transactions on Wireless Communications 2(4): 773-786.

Zhou, S., Wang, Z. \& Giannakis, G. (2005). Quantifying the Power Loss When Transmit Beamforming Relies on Finite-Rate Feedback, IEEE Transactions on Wireless Communications 4(4): 1948-1957.

Zhou, Z., Dong, Y., Zhang, X., Wang, W. \& Zhang, Y. (2004). A Novel Antenna Selection Scheme in MIMO Systems, International Conference on Communications, Circuits and Systems (ICCCAS 04), Vol. 1, pp. 190-194. 
Zhou, Z. \& Vucetic, B. (2004). MIMO Systems with Adaptive Modulation, Proc. of the 59th Vehicular Technology Conference (VTC 04), Vol. 2, pp. 765-769.

Zhuang, H., Dai, L., Zhou, S. \& Yao, Y. (2003). Low Complexity Per-Antenna Rate and Power Control Approach for Closed-Loop V-BLAST, IEEE Transactions on Communications 51(11): 1783-1787. 


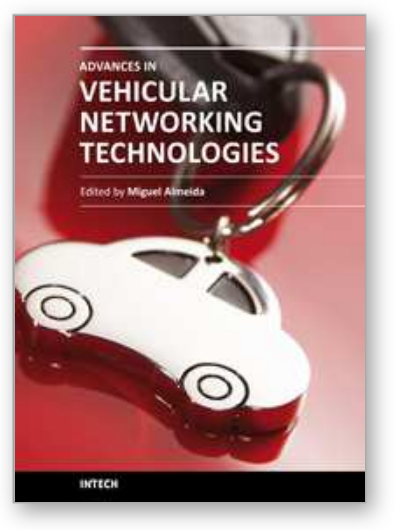

\author{
Advances in Vehicular Networking Technologies \\ Edited by Dr Miguel Almeida
}

ISBN 978-953-307-241-8

Hard cover, 432 pages

Publisher InTech

Published online 11, April, 2011

Published in print edition April, 2011

This book provides an insight on both the challenges and the technological solutions of several approaches, which allow connecting vehicles between each other and with the network. It underlines the trends on networking capabilities and their issues, further focusing on the MAC and Physical layer challenges. Ranging from the advances on radio access technologies to intelligent mechanisms deployed to enhance cooperative communications, cognitive radio and multiple antenna systems have been given particular highlight.

\title{
How to reference
}

In order to correctly reference this scholarly work, feel free to copy and paste the following:

Rami Abu-alhiga and Harald Haas (2011). Novel Co-Channel Interference Signalling for User Scheduling in Cellular SDMA-TDD Networks, Advances in Vehicular Networking Technologies, Dr Miguel Almeida (Ed.), ISBN: 978-953-307-241-8, InTech, Available from: http://www.intechopen.com/books/advances-in-vehicularnetworking-technologies/novel-co-channel-interference-signalling-for-user-scheduling-in-cellular-sdma-tddnetworks

\section{INTECH}

open science | open minds

\section{InTech Europe}

University Campus STeP Ri

Slavka Krautzeka 83/A

51000 Rijeka, Croatia

Phone: +385 (51) 770447

Fax: +385 (51) 686166

www.intechopen.com

\section{InTech China}

Unit 405, Office Block, Hotel Equatorial Shanghai

No.65, Yan An Road (West), Shanghai, 200040, China

中国上海市延安西路65号上海国际贵都大饭店办公楼 405 单元

Phone: +86-21-62489820

Fax: +86-21-62489821 
(C) 2011 The Author(s). Licensee IntechOpen. This chapter is distributed under the terms of the Creative Commons Attribution-NonCommercialShareAlike-3.0 License, which permits use, distribution and reproduction for non-commercial purposes, provided the original is properly cited and derivative works building on this content are distributed under the same license. 